\title{
SOIL CREEP AND CONVEX-UPWARD VELOCITY PROFILES: THEORETICAL AND EXPERIMENTAL INVESTIGATION OF DISTURBANCE-DRIVEN SEDIMENT TRANSPORT ON HILLSLOPES
}

\author{
JOSHUA J. ROERING* \\ Department of Geological Sciences, University of Oregon, Eugene, OR 97403-1272, USA
}

Received 25 September 2003; Revised 29 February 2004; Accepted 8 April 2004

\begin{abstract}
The movement of unconsolidated materials near the Earth's surface is often driven by disturbances that occur at a range of spatial and temporal scales. The nature of these disturbances ranges from highly variable, such as tree turnover, to periodic and predictable, such as frost heave or creep. To explore the effect of probabilistic disturbances on surface processes, we formulated a granular creep model with analogy to rate process theory (RPT) used for chemical reactions. According to the theory, individual particles must be energized to a height greater than adjacent particles in order for grain dilation and transport to occur. The height of neighbouring particles (which is akin to activation energy in chemical reactions) varies with slope angle such that energy barriers get smaller in the downslope direction as slopes steepen. When slopes approach the friction-limited angle of repose, the height of energy barriers approaches zero and grains flow in the absence of disturbance. An exponential function is used to describe the probability distribution of particle excitation height although alternative distributions are possible. We tested model predictions of granular dynamics in an experimental sandpile. In the sandpile, acoustic energy serves as the disturbance agent such that grains dilate and shear in response. Particle velocities are controlled by the frequency of energy pulses that result in grain displacement. Using tracer particles, we observed a convex-upward velocity profile near the surface of the sandpile, consistent with predictions of our RPT-based velocity model. In addition, we depth-integrated the velocity model to predict how flux rates vary with inclination of the sandpile and observed non-linear flux-gradient curves consistent with model predictions. By varying the acoustic energy level in the experimental sandpile, we documented changes in the rate of grain movement; similar changes in modelled velocities were achieved by varying the exponent of the particle excitation probability distribution. The general agreement between observed and modelled granular behaviour in our simple laboratory sandpile supports the utility of RPT-based methods for modelling transport processes (e.g. soil creep, frost heave, and till deformation), thus enabling us to account for the probabilistic nature of disturbances that liberate sediment in natural landscapes. Copyright @ 2004 John Wiley \& Sons, Ltd.
\end{abstract}

KEY WORDS: hillslope; soil creep; rate process theory; disturbances; granular dynamics

\section{INTRODUCTION}

Mechanisms by which soil and rock travel downslope determine the magnitude and frequency of sediment production but are often difficult to characterize and quantify. Since the 1960s, geomorphologists have performed numerous field-based monitoring studies to quantify rates of soil transport in the absence of overland flow over annual to decadal timescales (Young, 1963; Kirkby, 1967; Fleming and Johnson, 1975; Young and Rapp, 1978; Finlayson, 1981; Auzet and Ambroise, 1996; Yamada, 1997; Sasaki et al., 2000; Matsuoka, 2001; Heimsath et al., 2002). While some datasets have revealed continuous, convex-upward velocity profiles, others have revealed non-systematic results, owing to the stochasticity of transport mechanisms (Flavell, 1987; Clarke et al., 1999). As originally postulated by Davis (1892) and Gilbert (1909), many of these field studies suggest that downslope transport is driven by disturbances related to bioturbation (both faunal and floral), wet-dry and freeze-thaw cycles, mineral weathering, and other processes. Such disturbances effectively detach and mobilize sediments, allowing them to overcome resisting forces and be transported due to gravity.

\footnotetext{
* Correspondence to: J. J. Roering, Department of Geological Sciences, University of Oregon, Eugene, OR 97403-1272, USA. E-mail:
} jroering@uoregon.edu 
The spatial and temporal scale of sediment-mobilizing disturbances varies greatly between landscapes. In humid, forested areas with coarse granular soils, tree turnover, root growth, and small mammal burrowing may dominate soil transport and retard the development of soil horizons (Schaetzl et al., 1990). Such processes are highly stochastic, detaching and transporting packets of soil ranging in volume from $10^{1}$ to $10^{6} \mathrm{~cm}^{3}$ (Finlayson, 1985; Schaetzl, 1986). Quantification of soil transport by such 'macro-scale' disturbances has been limited (Black and Montgomery, 1991; Gabet, 2000; Heimsath et al., 2001; Roering et al., 2002; Gabet et al., 2003).

In the absence of macro-scale perturbations, soil displacement may be controlled by disturbances that occur at the particle scale. Moisture or temperature changes can cause soil particles to be mobilized relative to their neighbouring particles. The disturbance mechanism may penetrate to some depth producing a mobile layer that undergoes distributed downslope 'shearing'. In such cases, the velocity of particles near the surface reflects the cumulative movement of the underlying particles. Commonly, empirical datasets of soil creep, solifluction, and subglacial till deformation reveal velocity profiles with a characteristic convex-upward form, such that particle velocities are highest at the surface and decrease non-linearly with depth (Figure 1) (e.g. Eyles and Ho, 1970;
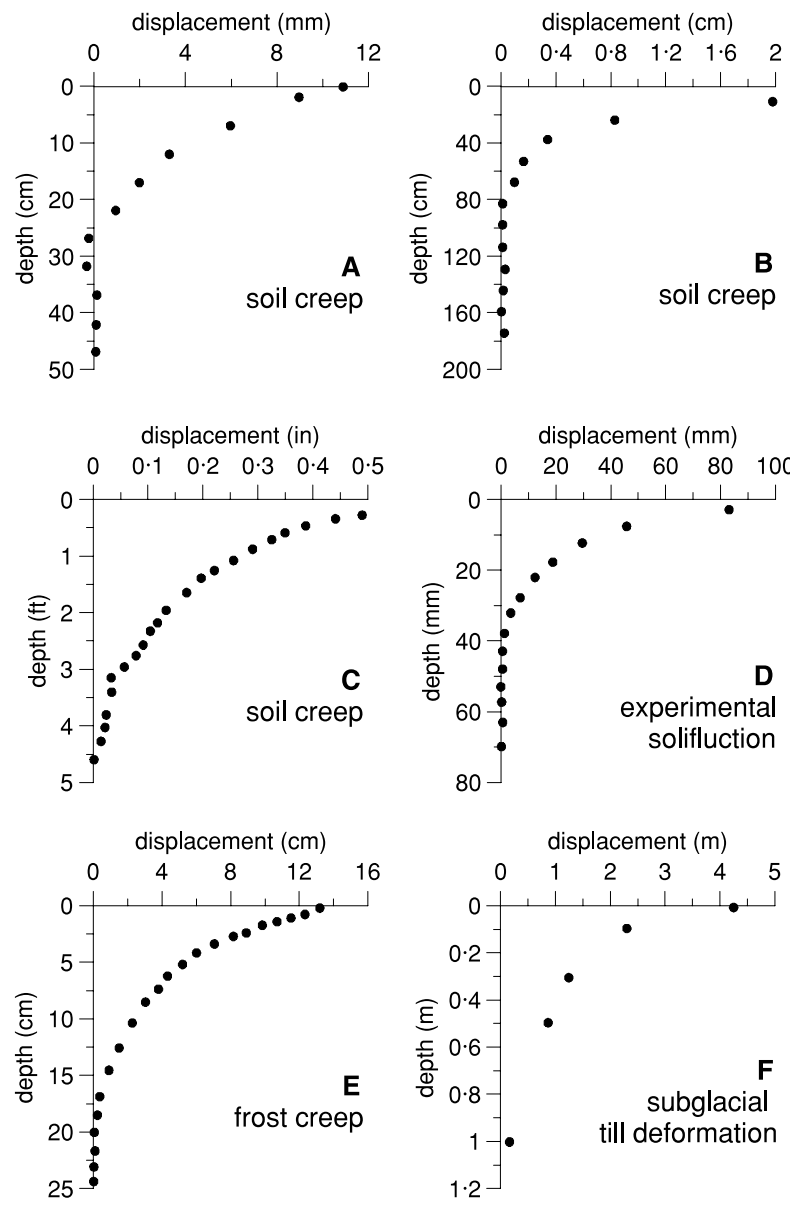

Figure 1. Displacement profiles exhibiting a characteristic convex-upward shape. Displacement patterns are associated with: (A) a freezethaw cycle (9 February - 4 March 1985) on a forested slope near Strasbourg, France (Auzet and Ambroise, 1996); (B) accumulation of wetdry cycles (1964 to 1969) in a clay-rich tropical soil, Kuala Lumpur, Malaysia (Eyles and Ho, 1970); (C) annual wet-dry cycle (19 March 1970 to 6 February 1971) in a silty clay soil, Stanford, California (Fleming and Johnson, 1975); (D) accumulation of seven freeze-thaw cycles in an experimental slope of sandy silt (Harris et al., 1997); (E) accumulation of freeze-thaw cycles (1992 to 1995) in sand and silt debris, Japanese Alps (Matsuoka, 1998); (F) accumulation of 17 days of subglacial till deformation, Breidamerkurjokull, Iceland (Boulton and Dobbie, 1998) 
Fleming and Johnson, 1975; Auzet and Ambroise, 1996; Harris et al., 1997; Boulton and Dobbie, 1998; Matsuoka, 1998). In alpine environments, seasonal frost heave activity displaces grains through the growth of ice crystals (Matsuoka, 1998), whereas in temperate regions with silty clay soils, seasonal wet-dry cycles can cause particle rearrangement and net downslope transport (Fleming and Johnson, 1975). Because the profiles shown in Figure 1 are associated with different disturbance mechanisms, soil properties and climatic regimes, convex-upward velocity profiles may be a general property of systems dominated by particle-scale grain movement.

In simulations at the landscape scale, details of the velocity-depth structure are often neglected and soil transport is modelled deterministically such that transport rate varies as a function of hillslope gradient or the product of gradient and average soil depth (e.g. Kirkby, 1985; Howard, 1994; Tucker and Bras, 1998; Braun et al., 2001; Anderson, 2002). Such approaches cannot easily account for how variability in disturbances and environmental characteristics regulate the rate of soil movement. Reasoning that the frequency of sedimentmobilizing disturbances is not uniform, but more likely exhibits a probability distribution, Culling (1983) adapted rate process theory (RPT) to simulate sediment transport processes. Rate process theory, which originated in the pioneering work on chemical reaction rates by Eyring (1935), dictates that atoms or molecules are constrained from movement by energy barriers separating adjacent equilibrium positions. Displacement (and thus chemical reaction) requires that atoms become thermally activated with energy of sufficient magnitude to surmount adjacent potential barriers (Mitchell, 1993). Using a series of laboratory experiments, Mitchell et al. (1968) applied RPT to quantify the deformation of clay-rich soils, wherein the primary barriers to particle movement were intermolecular or interatomic bonds. Culling (1983) recognized that RPT can be more generally applied at the grain scale such that a specific amount of mechanical energy is required to overcome frictional resistance and displace a grain or clast. In this conceptual framework, the net rate of downslope transport depends on the probability distribution of disturbance magnitude.

Here, we describe a novel soil transport theory based on the notion that clasts must become activated via disturbances in order for transport to occur. The goals of this study are to: (1) explore the conditions necessary to generate convex-upward velocity profiles; (2) incorporate probability into a general sediment transport relationship; and (3) test model predictions using experiments that represent a simplified version of real hillslopes. The proposed transport model, which is distinguished from Culling's (1983) application of RPT as described below, posits that particle movement is resisted by friction and thus is highly sensitive to hillslope angle, particularly as slopes approach the angle of repose. Velocity profiles calculated using the model are consistent with those shown in Figure 1, indicating that the interaction of frictional resistance and disturbances exhibiting a given probability distribution may conspire to produce convex-upward velocity-depth relationships. To parameterize and test the model, we performed a series of granular transport experiments in a laboratory sandbox in which disturbances were generated acoustically. Although this mechanism for generating grain movement is not directly akin to natural processes, the general correspondence between model predictions and experimental results supports the model's ability to represent disturbance-driven transport of unconsolidated particles. The approach described here is a departure from deterministic models and may allow us to better account for the probabilistic nature of processes that transport sediment near the Earth's surface.

\section{MODEL OF DISTURBANCE-DRIVEN TRANSPORT OF UNCONSOLIDATED MATERIALS}

We represent unconsolidated sediment with a simple geometric model that describes the physical barriers resisting transport. Soil particles are buttressed from downslope movement by their neighbouring particles, such that they must dilate over one another in order for transport to occur. Even 'loose' soils (as defined according to critical-state soil mechanics) typically experience a brief phase of dilation before undergoing contractive deformation (Terzaghi et al., 1996). In effect, our approach attempts to quantify the likelihood that a particular grain will shear relative to its neighbours. Although the probability of grain dilation and transport likely varies with density and grain size distribution, here we focus on the influence of gravitational loading and steepness. For the simple case of a uniform particle size distribution, particles must be energized (or activated) to a height proportional to the diameter of adjacent grains (Figure 2A). Following the convention of rate process theory (RPT) (Mitchell, 1993), the distribution of grain excitation heights, $h$, is represented with an exponential distribution: 

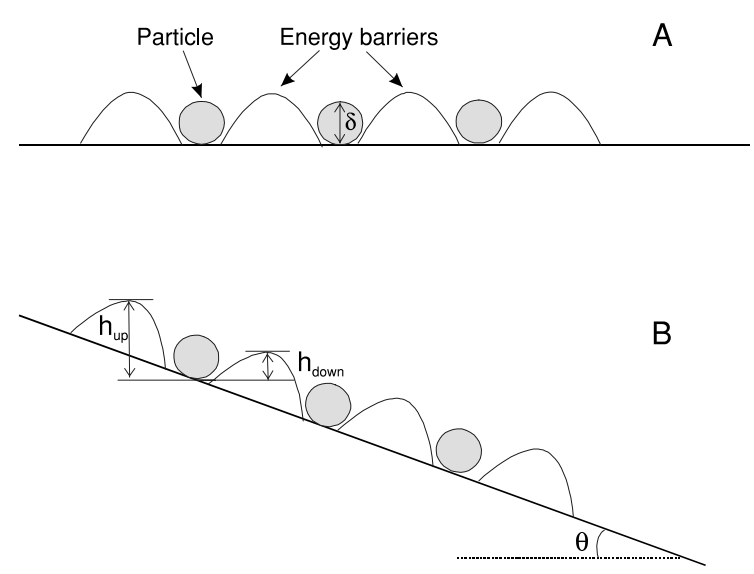

Figure 2. Schematic of model geometry at the particle level. (A) Mobilization of particles is impeded by mechanical barriers ('energy barriers'), which are in reality neighbouring particles. Grain dilation, shearing, and transport occur only when grains are energized to a height greater than adjacent barriers. (B) On an inclined slope, the height required for a particle to displace downslope decreases, whereas the height required for upslope travel increases. As the slope angle $(\theta)$ approaches the angle of internal friction $(\phi), h_{d o w n}$ approaches zero such that grain transport occurs in the absence of disturbances that energize or activate grains

$$
P[h] \propto e^{-\left(\frac{h}{h_{o}}\right)}
$$

where $h_{o}$ is the mean excitation height (in units of length, L). Thus, the probability, $P$, that a grain will be excited above the activation threshold and translate into an adjacent energy or potential well varies with the height of neighbouring particles according to:

$$
P\left[h>h_{*}\right] \propto e^{-\alpha h_{*}}
$$

where $h_{*}$ is the height of adjacent particles (L) and $\alpha=1 / h_{\mathrm{o}}$. Although other distributions may be used in place of Equations 1a and 1b, we use an exponential function because it is mathematically simple and many natural processes are well described by exponentials (such as the duration and intensity of rainfall events). Individual particles may be transported more than one particle diameter away from their original position, thus the cumulative probability of transport by excitation is given by:

$$
P_{\text {cum }}=P+P^{2}+P^{3}+\ldots
$$

where $P, P^{2}$, and $P^{3}$ are the probabilities of a particle moving one, two and three positions from the original particle position, respectively (where $P$ is calculated with Equation 1b). The following function corresponding to this infinite series allows us to analytically calculate the cumulative probability of particle motion:

$$
P_{\text {cum }}=\frac{P}{1-P}
$$

On flat terrain, the probability of grain displacement is equal in all directions (Figure 2A). In contrast, on a tilted slope, the activation height required to override upslope grains is greater than that for downslope grains (Figure 2B). As a result, the probability of downslope travel exceeds that of upslope travel. Using our simple geometric model, the height required to exceed downslope and upslope transport barriers, $h_{d o w n}$ and $h_{u p}$, is given by:

$$
h_{\text {down }}=\delta \sin (\phi-\theta)
$$




$$
h_{u p}=\delta \cos (90-\phi-\theta)
$$

where $\theta$ is the slope angle (degrees), $\phi$ is the coefficient of internal friction (degrees), and $\delta$ is grain diameter (L) (Figure 2B). If the slope angle equals or exceeds the angle of internal friction $(\theta=\phi)$, particles require zero energy to move downslope $\left(h_{\text {down }}=0\right)$ and freely dilate and displace downslope.

Particles near the surface require less excitation energy for movement than do deeper particles because of decreased overburden, contributing to rapid velocities near the surface. To quantify how the variation of increased overburden with depth influences particle activation energy, we posit that $\alpha$ (which is equal to $1 / h_{o}$ ) varies proportionally with lithostatic stress. Thus, $\alpha$ varies linearly with vertical depth, $d$, and depth measured normal to the surface, $y$, respectively, according to:

$$
\begin{gathered}
\alpha=\beta d \\
\alpha=\frac{\beta y}{\cos \theta}
\end{gathered}
$$

where $\beta$ is a constant $\left(\mathrm{L}^{-2}\right)$. By substitution, Equation 5a indicates that $h_{o}=1 /(\beta d)$, such that mean particle excitation height decreases non-linearly with depth. While another functional relationship in place of Equation 5 could be justified, we chose a simple model to minimize parameter and model complexity. When coupled, Equations $1 \mathrm{~b}$ and $5 \mathrm{a}$ state that with increasing depth, the exponent of the probability distribution becomes more negative such that the probability of particle displacement decreases.

Analogous to the formulation of Mitchell (1993), we define the net downslope particle velocity as proportional to the difference between the probability of downslope and upslope displacement. By combining Equations 1 and 3-5, the depth-dependent relative particle velocity, $v_{r}(y)$, can be calculated as the difference between the downslope and upslope displacement components, according to:

$$
v_{r}(y)=K\left[P_{\text {cum }(\text { down })}-P_{\text {cum }(u p)}\right]=K\left[\left(\frac{e^{-\beta D y}}{1-e^{-\beta D y}}\right)-\left(\frac{e^{-\beta U y}}{1-e^{-\beta U y}}\right)\right]
$$

where $K$ is a rate constant $\left(\mathrm{L} \mathrm{T}^{-1}\right), D=\delta \sin (\phi-\theta) / \cos \theta$, and $U=\delta \cos (90-\theta-\phi) / \cos \theta$. According to this simple formulation, three parameters characterize the velocity profile of creeping soils: $K$ scales the absolute transport rate, $\phi$ is the aspect ratio of energy barriers (equivalent to the internal angle of friction), and $\beta$ accounts for variations in the activation energy probability distribution. For the case of zero or negligible disturbance, $\beta=\infty$.

Equation 6 quantifies the velocity of particles relative to their neighbours. To obtain the total velocity at a point in the soil column, $v_{t}(y)$, we sum the velocity of underlying grains. Similar to the formulation by Iverson and Iverson (2001), the total velocity at a point is given by the integral:

$$
v_{t}(y)=-\frac{1}{\delta} \int_{\infty}^{y} v_{r}(y) d y
$$

Integrating Equation 7a, we obtain:

$$
v_{t}(y)=-\frac{K}{\beta \delta}\left(\frac{\ln \left(1-e^{-\beta D y}\right)}{D}-\frac{\ln \left(1-e^{-\beta U y}\right)}{U}\right)
$$

As $y$ approaches zero (i.e. at the surface), Equation $7 \mathrm{~b}$ indicates that particles attain infinite velocities. However, the shallowest depth for which particle velocity can be calculated (or even measured) is limited by particle size. Thus, the finite size of soil particles constrains the range of depths over which Equation $7 \mathrm{~b}$ can be applied. In landscapes where soils mantle bedrock or coherent substrate, the depth of mobile regolith limits the range of application. For this contribution, we assume that soil profiles are sufficiently deep and are not influenced by 
an underlying coherent substrate. Thus, Equation 7b is applied for $\delta<y<\infty$, where $\delta$ is the average particle diameter. For the experiments described below, the dimensions of the apparatus determine the depth for which particle velocities are calculated.

In addition, Equation $7 \mathrm{~b}$ can be used to quantify sediment flux. Sediment flux is the integral of sediment or particle velocity along a soil profile:

$$
q_{s}=-\frac{1}{\delta} \int_{\infty}^{\delta} \frac{K}{\beta}\left(\frac{\ln \left(1-e^{-\beta D y}\right)}{D}-\frac{\ln \left(1-e^{-\beta U y}\right)}{U}\right) d y
$$

Because a simple analytical expression for Equation 8 was elusive, we used a numerical integration algorithm (Press et al., 1992) to calculate how sediment flux varies according to model parameters and slope angle $(\theta)$.

This theoretical framework is constructed on the assumption that particles generally maintain frictional contact with their neighbours; excitations that induce movement are abbreviated and inertial forces are small. Thus, although our model does account for grain-scale instability as slopes approach the friction angle, it does not explicitly address discrete failures that initiate on steep slopes and transform into avalanches.

\section{MODEL PREDICTIONS OF VELOCITY AND FLUX RELATIONSHIPS}

Equation $7 \mathrm{~b}$ predicts that particle velocity is highest near the surface and decreases non-linearly with depth, giving a characteristic convex-upward form (Figure 3A). Velocities increase towards the surface because of the
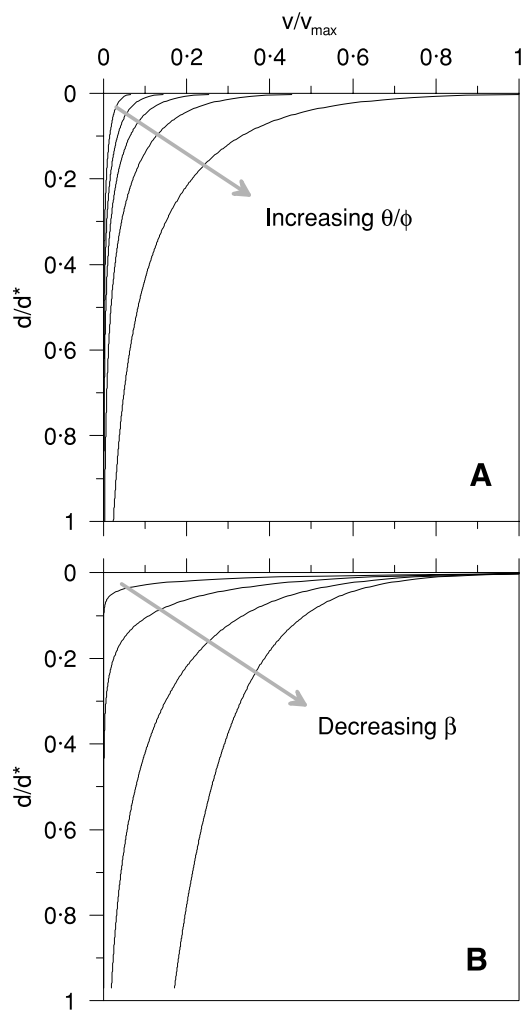

Figure 3. Variation of velocity profiles predicted using Equation $7 \mathrm{~b}$. Depth is normalized by $0 \cdot 2 \mathrm{~m}$ for both curves. (A) Velocity profile variation given changes in slope inclination (measured relative to the internal angle of friction), $\theta / \phi$. Values of $\theta / \phi$ are $0 \cdot 16,0 \cdot 32,0 \cdot 48,0 \cdot 64$ and $0 \cdot 80$, moving from left to right. Velocities increase non-linearly with incremental increases in slope angle and the depth of displacement also increases with slope. (B) Velocity profile variation given changes in the disturbance probability distribution parameter, $\beta$. With decreasing $\beta$, the magnitude of disturbance increases and the depth of transport increases producing broadly convex profiles 


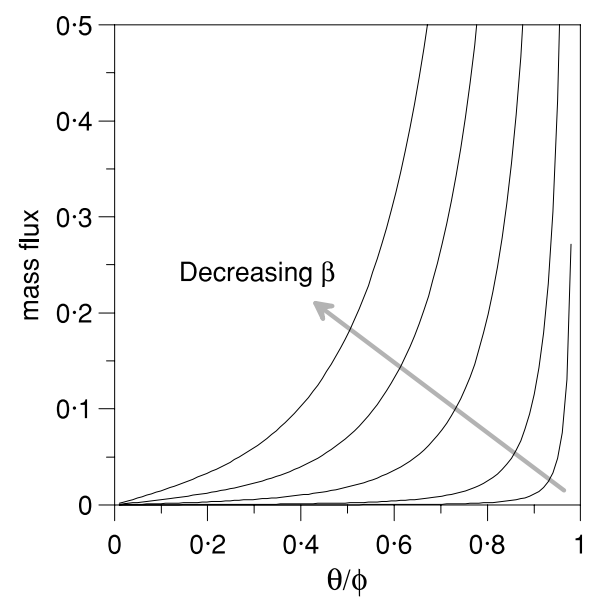

Figure 4. Variation of sediment mass flux with hillslope gradient (relative to the internal angle of friction), $\theta / \phi$. Curves reflect different values of $\beta$ such that decreasing $\beta$ gives higher flux rates for a given slope angle. Flux rates increase rapidly as slopes approach the angle of repose, consistent with recently proposed non-linear transport models (see text). As $\beta$ gets large, disturbances become insignificant and the flux-gradient curve approximates a vertical line at $\theta / \phi=1$

accumulation of underlying particle transport and increasing frequency of disturbance. We used Equation $7 \mathrm{~b}$ to calculate how the shape of velocity profiles varies with slope angle (which is normalized by $\phi$ ). For this calculation, we normalized the velocity for each of the curves (indicated as $v / v_{\max }$ ) by the maximum velocity calculated for the curve with the maximum value of $\theta / \phi$. Although the curves are calculated by linearly increasing the ratio $\theta / \phi$, the resulting velocity field shows a non-linear increase in velocity owing to the influence of rapidly decreasing frictional resistance as slopes approach the angle of repose (Figure 3A). In addition, with increasing $\theta / \phi$ the depth of creeping grains increases as the disturbance magnitude becomes increasing likely to overcome frictional resistance. Absolute velocity values scale in proportion to the value of $K$.

Variations in the velocity profile with the disturbance frequency parameter, $\beta$, are shown in Figure 3B. To isolate how $\beta$ affects the velocity-depth relationship, we normalized each velocity curve by the maximum velocity for that particular curve. With decreasing $\beta$, the magnitude of disturbance increases such that velocities at a given depth increase. Upon examining the morphology of the profiles, we observed that the 'kink' of the velocity curve is shallow and sharply defined for high values of $\beta$ and becomes broader and deeper as $\beta$ decreases (Figure 3B). Most generally, our calculations demonstrate that: (1) $\phi$ determines the rate at which particle velocities increase for a given change in slope angle; (2) $\beta$ determines the breadth and depth of the convex portion of the velocity profile; and (3) $K$ scales the absolute rate of sediment transport.

By solving Equation 8 for a range of hillslope angles, we calculated how sediment mass flux varies with hillslope gradient (Figure 4). For this calculation, we used normalized values of slope $(\theta / \phi)$. Model curves illustrate that flux rates increase non-linearly as slopes approach the angle of repose at which point flux becomes infinite. This characteristic behaviour is similar to recently proposed non-linear sediment transport models which include a mechanism by which fluxes increase rapidly when they approach a critical slope angle (Howard, 1994; Roering et al., 1999; Gabet, 2003). In the limiting case of $\beta \rightarrow \infty$, the flux-gradient curve approximates a vertical line at $\theta / \phi=1$. This represents the case of zero or negligible disturbance frequency such that flux rates are zero when $\theta<\phi$ and become infinite when $\theta=\phi$. With decreasing $\beta$, the location of the flux non-linearity shifts toward lower slope angles due to an increase in velocity and the depth of particle motion.

\section{EXPERIMENTAL DISTURBANCE-DRIVEN GRANULAR CREEP}

To test predictions of the RPT-based transport equations and calibrate model parameters, we performed a series of granular creep experiments in a laboratory sandpile subject to acoustic stimulation. More generally, we used the experimental apparatus to address the following question: What are the minimum conditions necessary to 
produce convex-upward velocity profiles? In other words, what is the simplest dynamical system capable of generating commonly observed convex-upward velocity profiles? Toward this goal, we constructed a laboratory hillslope of granular material, enclosed in a Plexiglas box $(120 \mathrm{~cm}$ long by $60 \mathrm{~cm}$ high by $10 \mathrm{~cm}$ wide) with open ends (Roering et al., 2001). We set the ratio of grain diameter to box width greater than 120 to negate boundary effects, such as grain arching (Grasseli and Herrmann, 1997). In the laboratory hillslope, resistance to transport results solely from frictional strength via inter-particle contacts such that we do not account for additional processes that bind soils in real landscapes (e.g. cohesion and root reinforcement).

Disturbances are required to displace grains on slopes below the angle of repose. In order to provide a consistent, repeatable, and efficient disturbance mechanism that reflects the character of disturbances envisaged in our model formulation, we vibrated our laboratory hillslope with noise from a large speaker coupled to the sandbox. This acoustic mechanism produced continuous shearing of grains near the sandpile surface. To minimize potential frequency bias in our sandbox, we used random acoustic noise with constant intensity to drive spatially uniform sediment transport along the hillslope. We used two different granular materials for the experiments: glass beads with mean diameter equal to $0.5 \mathrm{~mm}$ and friction angle $(\phi)$ approximately equal to $31^{\circ}$, and natural sand grains with mean diameter equal to $0.7 \mathrm{~mm}$ and $\phi$ approximately equal to $35^{\circ}$.

The vibration magnitude is small enough that grains maintain constant frictional contact with their neighbours. With the cessation of vibration, granular motion stops immediately, suggesting that inertial forces imparted on grains are small relative to gravitational and frictional forces acting near the sandpile surface. To track grains and quantify dynamics, we buried packets of blue-dyed glass beads along the sidewall of the sandbox. Figure 5 shows the configuration of blue grains before and after a $1.25 \mathrm{~s}$ pulse of noise on a $23^{\circ}$ slope of glass beads. Grain flow is approximately slope-parallel, with displacements highest at the sandpile surface and decreasing rapidly with depth (Figure 5B). Although the dominant direction of grain movement is downslope, dispersal of grains is high near the surface and decreases rapidly with depth, reflecting the variability of particle excitation through the sandpile.

To explore the ability of the RPT-based model to represent granular dynamics, we performed experiments at different slope angles and noise intensity settings. This approach enables us to test how model parameters included in Equations $7 \mathrm{~b}$ and 8 correspond to variations in sandpile dynamics.
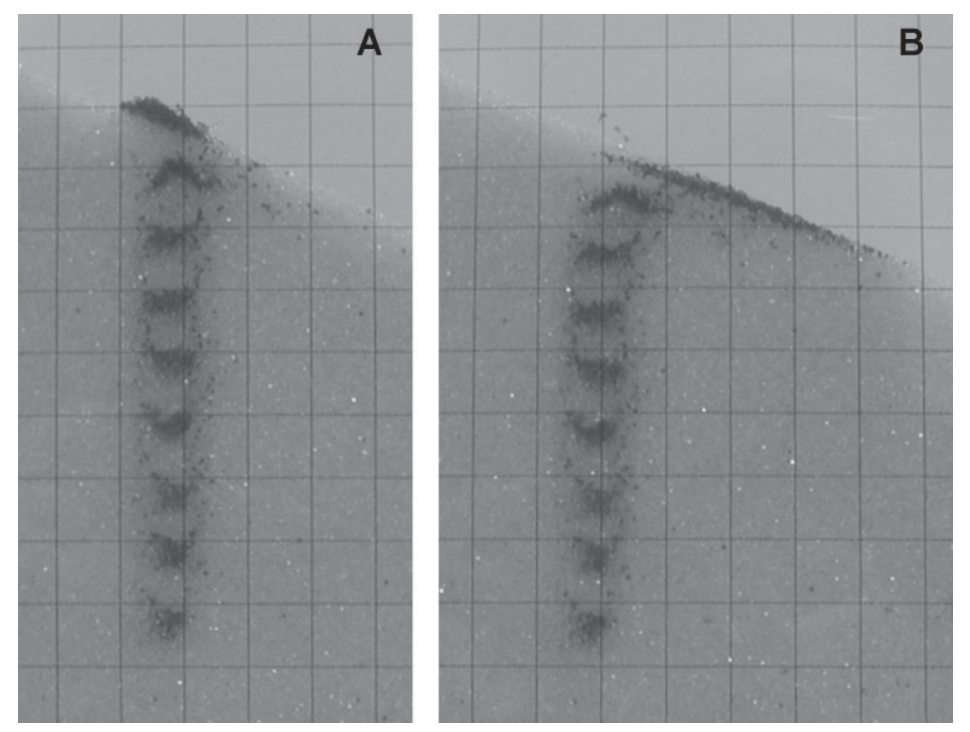

Figure 5. Photographic images of pre- and post-excitation grain configurations. Dark blue grains are buried in layers enabling quantification of displacement at different depths. In this experiment, glass beads (diameter $c .0 .5 \mathrm{~mm}$ and $\phi=31^{\circ}$ ) were subjected to acoustic stimulation for $1.25 \mathrm{~s}$. The overlying grid has horizontal and vertical spacing equal to $1 \mathrm{~cm}$. (A) Grain configuration before acoustic excitation. Nine grain packages are buried for use as markers. (B) Grain configuration following $1.25 \mathrm{~s}$ of excitation. Grains near the surface experience significant displacement and dispersal. Deeper grain units exhibit small displacements 


\section{EXPERIMENTAL AND MODELLED VELOCITY PROFILES}

To estimate grain velocities in our experimental hillslope, we digitized tracer grain positions using image analysis software. Digital photos of blue glass beads were taken before and after short pulses $(c .1 \mathrm{~s})$ pulses of noise. A grid with $1 \mathrm{~cm}$ spacing in the vertical and horizontal directions was positioned over the side of the sandbox enabling highly accurate characterization of grain positions. We buried discrete packets of grains through the upper $10 \mathrm{~cm}$ of the pile of beads enabling us to confidently distinguish the location of those grains from grain packages at different depths. Following image processing to remove photographic distortion, the positions of grains were digitized from before and after photos and repeat measurements showed that the mean position of grain packages (see Figure 5) was accurate to within 1-2 mm. Figure 6 illustrates the pre- and postexcitation configuration of six of the blue bead layers for the experiment shown in Figure 5. Because tracer beads are numerous, each data point we digitized represents $c .10-15$ grains as opposed to a single grain. To estimate velocities, we calculated the mean position of each layer before and after acoustic excitation and subtracted those values to obtain a displacement vector. We calculated velocity as the component of the displacement vector parallel to the sandpile surface divided by disturbance time. The depth of each velocity measurement was calculated by projecting the layer location normal to the sandpile surface. For most of our experiments, velocities were slope-parallel and deviated by less than $10^{\circ}$ from the orientation of the surface. We calculated the standard deviation of the grain layer locations and used Gaussian error propagation techniques to quantify the uncertainty of our velocity measurements.

We estimated velocity profiles using glass beads in our experimental hillslope for three different slope angles $\left(15^{\circ}, 20^{\circ}\right.$ and $\left.25^{\circ}\right)$ for low and high noise intensity settings $\left(0.03\right.$ and $\left.0.05 \mathrm{~W} \mathrm{~m}^{-2}\right)$. We consistently observed convex-upward velocity profiles in these experiments. Figure 7A shows the variation of velocity with depth for low-intensity noise; surface velocities on steep slopes $\left(25^{\circ}\right)$ are more than double those on slightly gentler slopes $\left(20^{\circ}\right)$. With increasing gradient, the kink of the velocity profile becomes broader and deeper, consistent with our RPT-based model predictions (Figure 3A). Estimates of the standard error in our velocity measurements

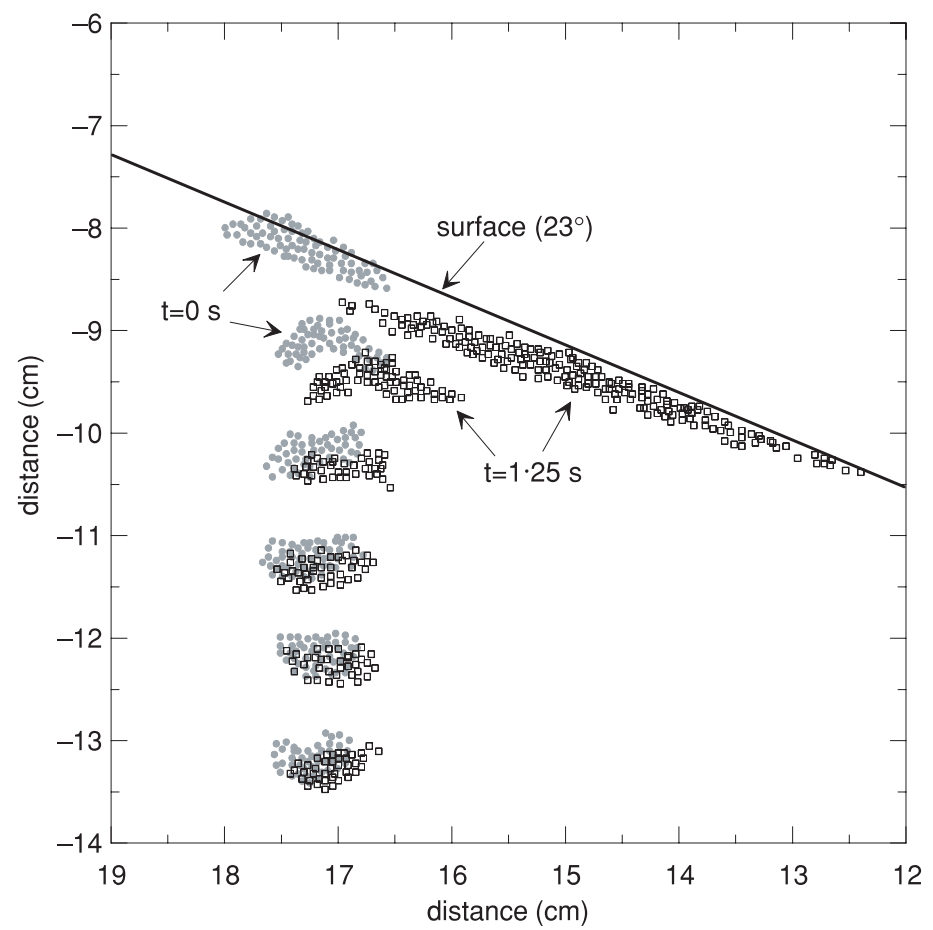

Figure 6. Plot of pre- and post-excitation grain configurations for the experiment illustrated in Figure 5. Closed grey circles and open squares indicate grain locations before and after acoustic excitation, respectively. Each digitized point represents the location of 10-15 actual grains, as they were too numerous to be individually located and digitized 

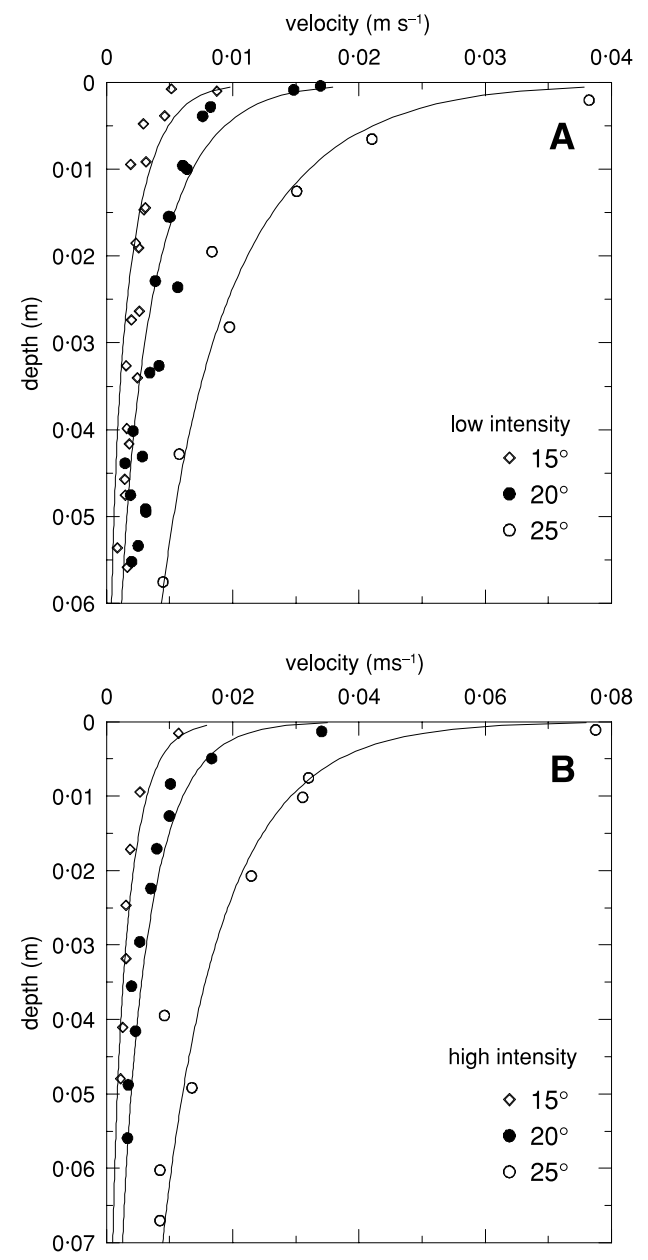

Figure 7. Experimental and modelled velocity profiles at different slope angles for low (A) and high (B) noise intensity experiments. Open diamonds, closed circles, and open circles represent the $15^{\circ}, 20^{\circ}$ and $25^{\circ}$ surface slopes, respectively, in both plots. Solid lines indicate model curves associated with proximal data points (Equation 7b). Both model and experimental data exhibit convex-upward behaviour. Calibration and validation procedures for the three-parameter $(K, \beta$ and $\phi)$ velocity profile model (Equation $7 \mathrm{~b})$ are described in the text. For both plots, $K=1.25 \times 10^{-4} \pm 0.4 \times 10^{-4} \mathrm{~m} \mathrm{~s}^{-1}$, and $\beta=1.27 \pm 0.2 \mathrm{~m}^{-2}$ and $\beta=0.85 \pm 0.15 \mathrm{~m}^{-2}$ for the low and high intensity experiments, respectively. The value for $\phi\left(31^{\circ}\right)$ was independently measured using a tilting table friction test

were typically on the order of 50 per cent; we omitted error bars from our plots to improve clarity. Nonetheless, it is important to recognize that velocity measurements near the surface are subject to significant uncertainty due to the rapid dispersion of grains at low confining stresses. Our experimental results for the high noise intensity case (Figure 7B) were similar except that absolute values of grain velocity were a factor of two greater than those for the low-intensity profiles.

Applying the three-parameter RPT-based transport model to our experimental results requires a parameterization strategy. As one end-member scenario, one could view each velocity profile independently and find the best-fit values of $\phi, \beta$, and $K$ (Equation $7 \mathrm{~b}$ ). This approach, however, fails to recognize that the laboratory velocity profiles are derived using the same granular material (which suggests that the value of $\phi$ should be constant) and the same noise source (which suggests that the rate of grain movement - represented by $K$ - should also be constant). In addition, this approach does not offer a useful test of the model's predictive ability. Furthermore, the value of $\phi$ can be estimated independently using tilting table friction tests (Lambe and Whitman, 1969). In other words, $\phi$ is determined outside the context of the model equations. Based on our laboratory friction tests, the glass beads used in these velocity profile experiments have $\phi=31 \pm 1^{\circ}$. 
We performed a test of the velocity profile model (Equation $7 \mathrm{~b}$ ) by calibrating the high angle $\left(25^{\circ}\right)$ experimental data for both intensity settings (see open circles in Figure 7) such that $K$ was forced to have a constant value for both profiles while the value of $\beta$ (which controls the magnitude of disturbances or granular excitation) was fitted independently for the low- and high-intensity datasets. For these two profiles, we used a non-linear curve-fitting algorithm to estimate that $K=1.25 \times 10^{-4} \pm 0.4 \times 10^{-4} \mathrm{~m} \mathrm{~s}^{-1}$, and $\beta_{\text {low }}=1.27 \pm 0.2 \mathrm{~m}^{-2}$ and $\beta_{\text {high }}=0.85 \pm 0.15 \mathrm{~m}^{-2}$ for the low- and high-intensity profiles, respectively (see solid lines in Figure 7). To test (or validate) the model predictions, we calculated velocity profiles for the lower angle $\left(15^{\circ}\right.$ and $\left.20^{\circ}\right)$ profiles using the fitted parameters (see solid lines associated with open diamonds and closed circles in Figure 7). The model exhibited promising predictive ability as the experimental data were consistently well represented by the model curves (Figure 7). In particular, the model curves successfully captured changes in the shape of the velocity profiles as well as the highly non-linear variation in velocity associated with changes in surface slope for both sets of experiments.

\section{EXPERIMENTAL AND MODELLED FLUX-GRADIENT RELATIONSHIPS}

We documented flux-gradient relationships in our experimental apparatus by establishing a steady-state flux regime and measuring the slope angle associated with different steady-state flux rates. Specifically, we used a hopper mounted above the sandbox to feed grains onto the top of the hillslope at a specified rate. With the addition of acoustic excitation and subsequent grain transport, the sandpile adjusted its surface slope to transport the flux supplied via the hopper. When the system reached steady state such that the flux of grains into and out of the sandbox was determined to be equal, we measured the associated slope angle (Roering et al., 2001). We quantified flux rate by collecting and weighing grains exiting the sandbox at given time intervals. By measuring the spatially uniform, steady surface slope associated with different supply rates, we determined the fluxgradient relationship. For these experiments, we used natural sand grains with mean grain diameter equal to $0.7 \mathrm{~mm}$ and internal angle of friction $(\phi)$ equal to $35 \pm 1^{\circ}$. We generated experimental flux-gradient curves for four different noise intensity settings $\left(0,0.01,0.03\right.$ and $\left.0.05 \mathrm{~W} \mathrm{~m}^{-2}\right)$.

The experimental flux-gradient curves are distinctly non-linear such that flux rates increase rapidly as gradient approaches a critical value (Figure 8). Similar to predictions of Equation 8 (Figure 4), the non-linearity becomes more distinct (or sharp) with decreasing excitation intensity. For a given gradient, flux rates increase monotonically

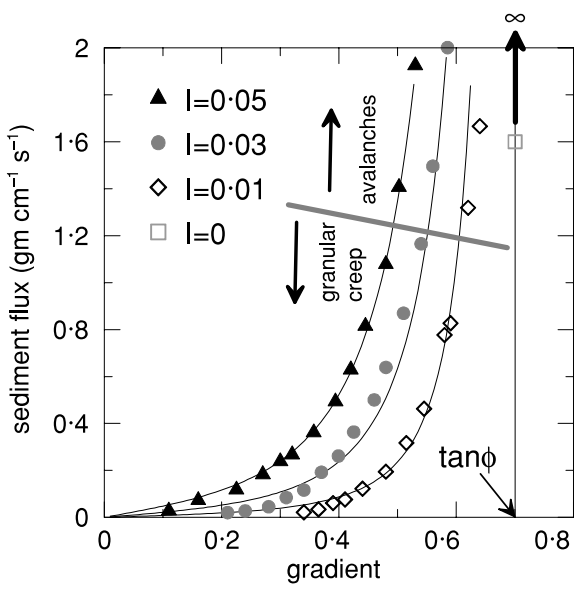

Figure 8. Experimental and modelled flux-gradient curves for different noise intensity settings. Closed triangles, close grey circles, open diamonds, and open squares represent empirical data derived for different levels of acoustic intensity from our speaker $(0 \cdot 05,0 \cdot 03,0 \cdot 01$ and $0 \mathrm{~W} \mathrm{~m}^{-2}$, respectively). Solid lines indicate model curves fitted to the group of proximal data points (Equation 8). Model and experimental data indicate that flux rates increase rapidly as slope angles approach a critical value. We calibrated the model curves using $\mathrm{K}=0.01 \mathrm{~m} \mathrm{~s}^{-1}$ and $\beta$ equal to $0.8,1.2,2.0$ and $\infty$ for the intensity levels of $0.05,0.03,0.01$ and $0 \mathrm{~W} \mathrm{~m}^{-2}$, respectively. The internal angle of friction for the natural sand used in these experiments was independently derived $\left(\phi=35^{\circ}\right)$. As the intensity setting approaches zero (no acoustic stimulation), the flux-gradient curve becomes a vertical line at $\theta=\phi$. Grain avalanches only occur on steep slopes and are not required to generate the non-linearity of the flux-gradient curve 
with increasing noise intensity. As intensity decreases, the sandpile exhibited imperceptible flux rates at a finite gradient. In other words, lower levels of acoustic excitation became increasingly incapable of dilating and shearing grains at a particular slope angle. Increased noise intensity decreased the angle at which transport was measurable. For the case with the highest intensity (see filled triangles in Figure 8) rates became difficult to estimate on slope gradients less than 0.1. For the case without acoustic stimulation (open squares in Figure 8), flux rates were zero when slopes angles $(\theta)$ were below the internal angle of friction $(\phi)$. As predicted by Equation 8, when $\theta=\phi$, flux rates approach infinity. For all the experiments shown in Figure 8, granular creep (defined as particle-by-particle dilation) was the dominant transport process. Only on extremely steep angles $(\theta \geq 0.9 \phi)$ did grain avalanching ensue.

To calibrate the experimental flux curves with Equation 8, we constrained $K$ to one value for all four curves and used the independently estimated value of $\phi=35^{\circ}$. From our non-linear calibration procedure, we estimated $K$ equal to $0.01 \mathrm{~m} \mathrm{~s}^{-1}$ and $\beta$ equal to $0 \cdot 8,1 \cdot 2,2 \cdot 0$, and $\infty$ for the $0.05,0 \cdot 03,0 \cdot 01$, and $0 \mathrm{~W} \mathrm{~m}^{-2}$ noise intensity experiments, respectively (model curves are shown as solid lines in Figure 8). The experimental curves are well represented by the model, particularly considering the conditions we imposed on the calibration. The model curve for the experimental data with $\mathrm{I}=0.03$ exhibits a systematic misfit with the data, slightly overpredicting fluxes at low gradients and underpredicting them at intermediate slopes (see filled grey circles in Figure 8). This may result from uncertainty in laboratory measurements of flux or slope angle or may reflect the inability of the model to properly account for very low transport rates at low slope angles. The model does not include a threshold of motion for low gradients, but instead indicates that the probability of grain dilation and transport becomes exceedingly small at low angles. In fact, this characteristic of the model appears to be consistent with our observations of very infrequent grain dilation along the surface of low-angle slopes.

\section{DISCUSSION}

The model proposed here offers a simple representation of how particle-scale disturbances integrate to produce convex-upward velocity profiles in unconsolidated sediments. Our model does not account for numerous complexities that may affect particle dynamics in natural systems, such as surface tension, density contrasts, soil moisture, weathering reactions, and particle size variations. Although our conceptual formulation of potential barriers resisting grain motion is analogous to the tenets of rate process theory, our model does not consider chemical bonding, which is the foundation of RPT (Eyring, 1935). Instead, our simplified derivation considers physical barriers to particle displacement such that the mechanical aspects of particle interaction are most relevant. The general pattern of convex-upward velocity profiles appears to be a fundamental feature of the proposed model. The pattern of velocity increasing toward the surface results for two reasons: (1) with decreasing depth particles accumulate displacement due to the motion of underlying particles; and (2) increased overburden decreases the mean particle activation height with depth. While this latter component of our model draws upon physical arguments in concept only, the generation of convex velocity profiles is not unique to our choice of how the mean particle excitation height varies with depth. Alternative models for the excitation probability distribution (Equation 1) and its variation with depth (Equation 5) are possible and may generate similar results.

Once calibrated, the velocity profile model proposed here provided accurate predictions of experimentally derived profiles generated at different slope angles (see Figure 7). This result was encouraging considering the complex nature of how the profiles varied for different slopes. Variation in the depth and breadth of the kink that defines the convex portion of the profiles was well represented by model predictions. Although conceptually intuitive, the physical significance of the two calibrated parameters $(K$ and $\beta)$ is difficult to quantify independently. The value of $K$ could be derived by consideration of particle diameter (which scales their travel distance) relative to the frequency of the disturbance source (which is related to the frequency spectrum of noise in our experiments). The parameter $\beta$ reflects how increasing overburden influences mean particle excitation height such that consideration of grain-scale stress perturbations as related to the lithostatic load (Iverson and Iverson, 2001) may prove to be useful for predictive purposes.

Observations of how granular dispersion varies with depth in our experiments may provide some insight regarding the value of $\beta$. Disturbances not only dilate grains and transport them downslope but also tend to disperse them along their path. As shown in Figure 6, the initially bean-shaped package of grains near the 

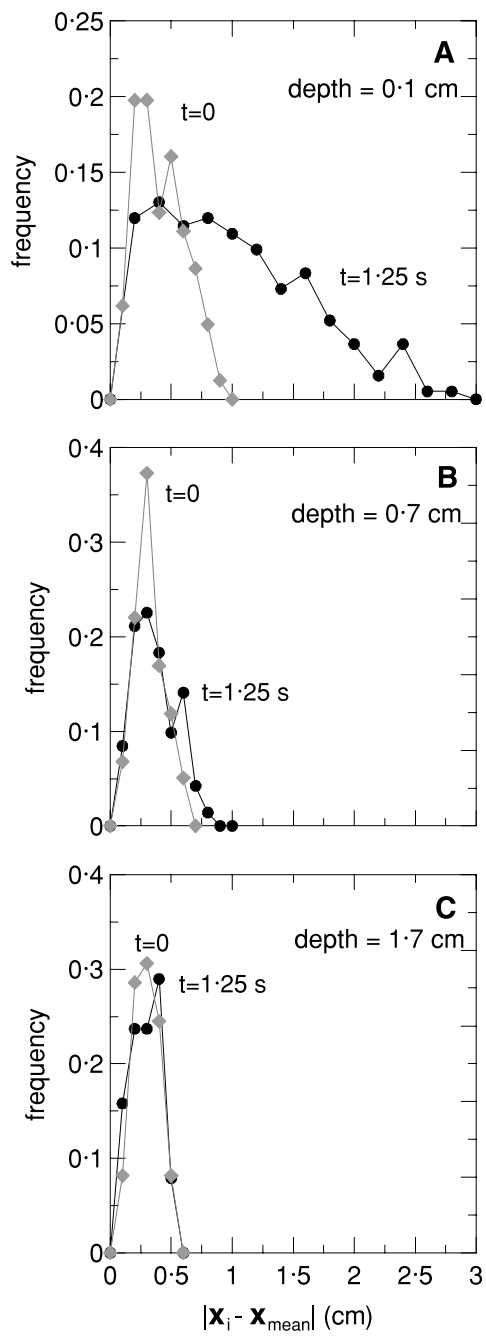

Figure 9. Histograms of grain dispersion following acoustic-driven transport as illustrated in Figure 5. Each histogram shows the distribution of particle distance from the mean location of that particular layer of grains. The top three layers of blue glass beads are shown here, corresponding to depths of $0 \cdot 1,0.7$ and $1.7 \mathrm{~cm}$, respectively. Open diamonds and closed circles show the distribution of particle distance prior to and following acoustic stimulation, respectively. Grain dispersion is strong near the surface and decreases non-linearly with depth

surface became stretched and elongate following the pulse of acoustic stimulation. This suggests that the velocity of particles was highly variable despite our representation of an average velocity at that depth. The range of particle velocities (or displacements) may reflect the shape of the disturbance probability distribution because the observed dispersion results from variability in granular response to disturbances. To explore this connection, we calculated how the dispersion of particles varies with depth for the dataset shown in Figure 6. For each layer of grains, we calculated the distance between each particle (whose location is represented as a vector, $\mathbf{x}_{\mathbf{i}}$ ) and the mean location of that layer $\left(\mathbf{x}_{\text {mean }}\right)$ before and after acoustic stimulation. Figure 9 shows the pre- and postexcitation distributions of $\left|\mathbf{x}_{\mathrm{i}}-\mathbf{x}_{\text {mean }}\right|$ for the upper three grain layers (depths equal to $0 \cdot 1,0.7$ and $1.7 \mathrm{~cm}$ ). At each depth, the initial distribution is relatively narrow with a mean value of less than $0.5 \mathrm{~cm}$. Following the noise pulse of $1.25 \mathrm{~s}$, the distribution of distances for the surficial layer (depth $=0.1 \mathrm{~cm}$ ) exhibited significant spreading with several grains more than $2.5 \mathrm{~cm}$ from the mean layer position (Figure 9A). Post-excitation dispersion is significantly decreased in the underlying layers as the distribution for depth $=0.7 \mathrm{~cm}$ shows minor, but perceptible spreading whereas dispersion at depth $=1.7 \mathrm{~cm}$ is indistinguishable based on this analysis. These results show a non-linear decrease in grain dispersion with depth similar to the non-linear decrease in the mean 
particle excitation height postulated in our model formulation (Equation 5). Relating $\beta$ to natural processes will require consideration of fluctuations in soil moisture, temperature, bioturbation, and other factors akin to those addressed by Kirkby (1995) and Matsuoka (1998).

The flux-gradient relationships predicted by Equation 8 are consistent with recent theories and datasets which indicate that in the absence of overland flow, sediment flux increases non-linearly as slope angles approach a critical value (Anderson, 1994; Howard, 1994; Roering et al., 1999; Gabet, 2000). The experimental analysis presented expands upon Roering et al. (2001), in that the influence of disturbance magnitude is included as a model parameter. Whereas the two-parameter non-linear transport model described in Roering et al. (1999) indicates that flux rates vary proportionally with gradient for low slopes, Equation 8 predicts an approximately linear flux-gradient relationship only when the disturbance magnitude is sufficiently high (low values of $\beta$ ). The model proposed here does not refute or contradict previous non-linear transport models' ability to represent transport in real landscapes (in particular, equation 8 in Roering et al., 1999), but instead enables us to consider more specifically how disturbance magnitude affects sediment transport processes in a particular regime. As our experiments demonstrate, disturbance of granular material generates distinctly non-linear flux-gradient relationships that are well described by our simplified RPT-based model.

In our experimental sandpile, grain avalanches occur on steep slopes (see Figure 8), although our theoretical model does not address this process. Grain avalanches in the experimental apparatus initiate along discrete failure surfaces several grain diameters below the surface. The destabilized mass becomes 'fluidized' and rapidly translates downslope, attenuating along its path. The mechanics of this dynamic process is beyond the scope of our model formulation.

In contrast to previous models for the velocity profile of soil creep (Culling, 1963; Kirkby, 1967), the model presented here includes a component describing the limit to frictional strength and thus accounts for rapid increases in velocity as slopes approach a critical value. Our approach of considering the frequency distribution of disturbances that drive particle movement is akin to that proposed by Culling (1963). He offers an elegant treatment of particle displacement resulting from the tradeoff between disturbance frequency and available void space in the regolith mantle, although the variation of velocity with depth is not considered. Culling (1963) posits that the distribution of displacement lengths (which may reflect upslope or downslope movement) is affected by pore space and gravity such that the distribution becomes skewed in the downslope direction when slope angles are non-zero or when ample void space is present. Likening soil transport to Brownian motion and borrowing theory from the physics of gases, Culling (1963) derives an analytical expression suggesting that the absolute transport rate of soil is related to the mean value of the frequency distribution of particle displacements. This conceptual framework also accounts for the dispersion of particles, which is difficult to quantify in nature, but was observed to a varying extent in our experiments. Recently, Heimsath et al. (2002) used cosmogenic radionuclides and optically stimulated luminescence analyses to test a probabilistic creep model similar to that proposed by Culling (1963). Their data indicate that particles throughout the profile 'visit' the surface such that the frequency of disturbance decreases with depth.

The theory derived by Kirkby (1967), which expands upon the classical work of Davison (1889), suggests that shear displacements in the soil mantle are proportional to the frequency of freeze-thaw events and the expansion coefficient for soil. At a particular depth, overburden overcomes the ability of disturbances to mobilize grains such that velocities approach zero. In contrast, our model does not explicitly determine the depth at which particle motion ceases; instead, the probability of motion becomes exceedingly small, such that any particle motion would be imperceptible. In fact, the depth to which our model can be applied will often be limited by underlying bedrock or coherent substrate. Less relevant to this contribution are studies of rate process theory applied to soil creep in the engineering literature (e.g. Feda, 1989), which typically address settings with extremely high loads and consider intermolecular bonding forces between clay particles (Mitchell et al., 1968).

Application of our proposed model to real landscapes will require field characterization of the scale and frequency of disturbances driving mass transport in addition to well-constrained observations of particle displacement. Our experiments illustrate the challenge of documenting transport in near-surface environments from sparsely sampled data; grain dispersion can significantly influence estimated displacements, which suggests that the use of multiple markers or particle tracers can improve measurements. Regarding the application of Equations $7 \mathrm{~b}$ and 8 to a specific field setting, the relative regularity of disturbances by freeze-thaw activity and 
associated moisture changes suggests that regions subject to solifluction may be a fruitful testing ground (e.g. Kirkby, 1995; Harris et al., 2001; Matsuoka, 2001). Finally, our model predicts velocity profiles similar to those generated by a recently proposed, physically based Coulomb friction model for subglacial till deformation (Iverson and Iverson, 2001). As stated by Iverson and Iverson (2001), independent prediction of field-based data using their model (or that proposed here) requires further characterization of processes related to model parameters as well as natural complexities.

\section{CONCLUSION}

Despite the simplistic underpinnings of its formulation, our proposed model predicts convex-upward sediment velocity profiles consistent with those observed in diverse field settings. By accounting for the stochastic nature of disturbances that overcome mechanical impediments to particle movement, the model enables us to better represent stochastic processes such as freeze-thaw and wet-dry cycles and bioturbation. Experiments using acoustically stimulated granular dynamics exhibit velocity profiles similar to those predicted by the theoretical model. Once calibrated, the model accurately predicted the magnitude and form of highly non-linear experimental velocity profiles generated for different slope angles. Although our model test was conducted on a simple, uniform grain size system, our results suggest that the model may be applied to represent particle (or sediment) dynamics that result from the interaction of: (1) friction-dominated resistance, and (2) disturbances of a given magnitude and frequency. Because these two processes control the transport of sediment in numerous natural environments, this approach may have broad application.

\section{ACKNOWLEDGEMENTS}

The author thanks K. Cuffey for a fruitful and informative conversation about rate process theory applied to soil mechanics, R. Iverson for insightful analyses and observations regarding granular experiments, and J. Kirchner for helpful suggestions regarding probability and model calibration. M.J. Kirkby provided thoughtful and useful comments that have improved the conceptual integrity and clarity of the manuscript. In addition, L. Sklar aided in the construction of the experimental apparatus and E. Montanaro performed numerous experimental analyses with care and patience. The author also thanks third-floor occupants of Cascade Hall for tolerating numerous high-decibel episodes of 'acoustic stimulation'. This work was partially funded by the Department of Geological Sciences, University of Oregon.

\section{REFERENCES}

Anderson RS. 1994. Evolution of the Santa Cruz Mountains, California, through tectonic growth and geomorphic decay. Journal of Geophysical Research 99(10): 20,161-20,174.

Anderson RS. 2002. Modeling the tor-dotted crests, bedrock edges, and parabolic profiles of high alpine surfaces of the Wind River Range, Wyoming. Geomorphology 46: 35-58.

Auzet AV, Ambroise B. 1996. Soil creep dynamics, soil moisture and temperature conditions on a forested slope in the granitic Vosges mountains, France. Earth Surface Processes and Landforms 21(6): 531-542.

Black TA, Montgomery DR. 1991. Sediment transport by burrowing animals, Marin County, California. Earth Surface Processes and Landforms 16: 163-172.

Boulton GS, Dobbie KE. 1998. Slow flow of granular aggregates: the deformation of sediments beneath glaciers. Philosophical Transactions of the Royal Society of London Series a-Mathematical Physical and Engineering Sciences 356(1747): 2713-2745.

Braun J, Heimsath AM, Chappell J. 2001. Sediment transport mechanisms on soil-mantled hillslopes. Geology 29: 683-686.

Clarke MF, Williams MAJ, Stokes T. 1999. Soil creep: Problems raised by a 23 year study in Australia. Earth Surface Processes and Landforms 24(2): 151-175.

Culling WEH. 1963. Soil creep and the development of hillside slopes. Journal of Geology 71(2): 127-161.

Culling WEH. 1983. Rate process theory in geomorphic soil creep. In Rainfall Simulation, Runoff and Soil Erosion, de Ploey J (ed.). Catena Suppl. Catena Verlag: Braunschweig; 191-214.

Davis WM. 1892. The convex profile of badland divides. Science 20: 245

Davison C. 1889. On the creeping of the soil cap through the action of frost. Geological Magazine 6: 255.

Eyles RJ, Ho R. 1970. Rates of seasonal creep of silty clay soil. Quarterly Journal of Engineering Geology 8: 1-29.

Eyring H. 1935. The activated complex and the absolute rate of chemical reactions. Chemical Review 17: 65-77.

Feda J. 1989. Interpretation of creep of soils by rate process theory. Geotechnique 39(4): 667-677.

Finlayson B. 1981. Field measurements of soil creep. Earth Surface Processes and Landforms 6: 35-48. 
Finlayson BL. 1985. Soil creep: a formidable fossile of misconception. In Geomorphology and Soils, Richards KS, Arnett RR, Ellis S (eds). George Allen \& Unwin: London; 141-158.

Flavell WS. 1987. Field investigation of a stochastic theory of soil creep. In International Geomorphology 1986; Proceedings of the First International Conference on Geomorphology; Part I, Gardiner V (ed.). John Wiley \& Sons: Chichester; 513-526.

Fleming RW, Johnson AM. 1975. Rates of seasonal creep of silty clay soil. Quarterly Journal of Engineering Geology 8: 1-29.

Gabet EJ. 2000. Gopher bioturbation: Field evidence for non-linear hillslope diffusion. Earth Surface Processes and Landforms 25(13): $1419-1428$.

Gabet EJ. 2003. Sediment transport by dry ravel. Journal of Geophysical Research - Solid Earth 108(B1): art. no. 2049.

Gabet EJ, Reichman OJ, Seabloom EW. 2003. The effects of bioturbation on soil processes and sediment transport. Annual Review of Earth and Planetary Science 31: 249-273.

Gilbert GK. 1909. The convexity of hilltops. Journal of Geology 17(4): 344-350.

Grasseli Y, Herrmann HJ. 1997. On the angles of dry granular heaps. Physica A 246: 301-312.

Harris C, Davies MCR, Coutard JP. 1997. Rates and processes of periglacial solifluction: An experimental approach. Earth Surface Processes and Landforms 22(9): 849-868.

Harris C, Rea B, Davies M. 2001. Scaled physical modelling of mass movement processes on thawing slopes. Permafrost and Periglacial Processes 12(1): 125-135.

Heimsath AM, Dietrich WE, Nishiizumi K, Finkel RC. 2001. Stochastic processes of soil production and transport: Erosion rates, topographic variation and cosmogenic nuclides in the Oregon Coast Range. Earth Surface Processes and Landforms 26(5): 531-552.

Heimsath AM, Chappell J, Spooner NA, Questiaux DG. 2002. Creeping soil. Geology 30: 111-114.

Howard AD. 1994. A detachment-limited model of drainage basin evolution. Water Resources Research 30(7): 2261-2285.

Iverson NR, Iverson RM. 2001. Distributed shear of subglacial till due to Coulomb slip. Journal of Glaciology 47(158): 481-488.

Kirkby MJ. 1967. Measurement and theory of soil creep. Journal of Geology 75(4): 359-378.

Kirkby MJ. 1985. A model for the evolution of regolith-mantled slopes. In Models in Geomorphology, Woldenberg MJ (ed.). Allen \& Unwin: London; 213-237.

Kirkby MJ. 1995. A model for variations in gelifluction rates with temperature and topography - Implications for global change. Geografiska Annaler 77A(4): 269-278.

Lambe TW, Whitman RV. 1969. Soil Mechanics. Series in soil engineering. Wiley: New York.

Matsuoka N. 1998. The relationship between frost heave and downslope soil movement: Field measurements in the Japanese Alps. Permafrost and Periglacial Processes 9(2): 121-133.

Matsuoka N. 2001. Solifluction rates, processes and landforms: a global review. Earth-Science Reviews 55(1-2): 107-134.

Mitchell JK. 1993. Fundamentals of Soil Behavior. John Wiley \& Sons: New York.

Mitchell JK, Campanella RG, Singh A. 1968. Soil creep as a rate process. Journal of the Soil Mechanics and Foundations Division, ASCE 94: 231-253.

Press WH, Teukolsky SA, Vetterling WT, Flannery BP. 1992. Numerical Recipes in C: The Art of Scientific Computing. Cambridge University Press: Cambridge.

Roering J, Almond P, McKean J, Tonkin P. 2002. Soil transport driven by biological processes over millenial timescales. Geology 30: 11151118.

Roering JJ, Kirchner JW, Dietrich WE. 1999. Evidence for nonlinear, diffusive sediment transport on hillslopes and implications for landscape morphology. Water Resources Research 35(3): 853-870.

Roering JJ, Kirchner JW, Sklar LS, Dietrich WE. 2001. Hillslope evolution by nonlinear creep and landsliding: An experimental study. Geology 29: 143-146.

Sasaki Y, Fujii A, Asai K, Tanaka K. 2000. Soil creep process and its role in debris slide generation; field measurements on the north side of Tsukuba Mountain in Japan. Engineering Geology 56(1-2): 163-183.

Schaetzl RJ. 1986. Complete soil profile inversion by tree uprooting. Physical Geography 7(2): 181-189.

Schaetzl RJ, Burns SF, Small TW, Johnson DL. 1990. Tree uprooting: review of types and patterns of soil disturbance. Physical Geography 11(3): 277-291.

Terzaghi K, Peck RB, Mesri G. 1996. Soil Mechanics in Engineering Practice. John Wiley \& Sons: New York.

Tucker GE, Bras RL. 1998. Hillslope processes, drainage density, and landscape morphology. Water Resources Research 34: $2751-2764$.

Yamada S. 1997. Seasonal variation in soil creep on a forested hillslope near Sapporo, Hokkaido, northern Japan. Chikei=Transactions Japanese Geomorphological Union 18(2): 117-130.

Young A. 1963. Soil movement on slopes. Nature 200(4902): 129-130.

Young A, Rapp A. 1978. A twelve-year record of soil movement on a slope. Zeitschrift fur Geomorphologie 29: 104-110. 